\title{
EL FENÓMENO CROSS-LICENSING EN EL DERECHO ESPAÑOL DE PATENTES
}

\author{
THE CROSS-LICENSING PHENOMENON IN THE SPANISH PATENT LAW
}

\author{
Luz Sánchez García
}

Doctora Europea en Derecho por la Universidad de Murcia. Especialista Universitario en Patentes y Transferencia de Tecnología (Magister Lvcentinvs). Profesora de Derecho Mercantil e Internacional Privado en Universidad Católica de Murcia.

E-mail: mdllsanchez@ucam.edu

Convidada

RESUMEN: Muchos resultados de investigación cristalizan en invenciones susceptibles de protección por un derecho de exclusiva. Puesto que el conocimiento es un continuum, es posible que para una adecuada explotación de una invención sea necesario acudir a otra anterior protegida por patente. Es aquí donde la licencia de explotación de patente cobra relevancia ya que viene a eliminar esa barrera a la innovación. Sin embargo, como contrapartida, en estas situaciones de dependencia, se podrá estipular una licencia recíproca sobre el nuevo resultado, dando así paso al fenómeno conocido como cross-licensing o licencias cruzadas. En todo caso, esta situación no sólo tiene cabida en escenarios puramente normativos (licencias obligatorias) sino también en otros donde impera la autonomía de la voluntad (licencias contractuales), quedando incluidos en esta esfera los llamados patent pools.

Palabras clave: Patentes dependientes, Licencias cruzadas, Licencias obligatorias, Consorcios de patentes.

ABSTRACT: Many researches crystallize in inventions that can be protected by an exclusive right. Since knowledge is a continuиm, for an adequate exploitation of an invention protected by patent may be necessary to explote another previous invention. This is where licensing becomes relevant as it eliminates that potencial barrier to innovation. However, as a counterpart, in these situations of dependency, a reciprocal license may be stipulated on the new result, thus giving rise to the phenomenon known as cross-licensing. This situation occurs not only in purely normative scenarios (compulsory licenses) but also within voluntary agreements (contractual licenses) including, then, the so-called patent pools.

Keywords: Dependent patents, Cross-licensing, Compulsory licensing, Patent pools

SUMÁRIO: Introducción; 1. Concepto y fundamento de las licencias cruzadas; 2. Supuestos de licencias cruzadas contemplados en la ley 24/2015, de 24 de julio, de patentes; 2.1. Licencia obligatoria por razones de dependencia. posibilidad de Cross-Licensing; 2.2. Acuerdos voluntarios de Cross-Licensing. especial atención a los acuerdos "Pool" de patente; 3. Principales ventajas e inconvenientes de los acuerdos voluntarios de Cross-Licensing; Fuentes. 


\section{INTRODUCCIÓN}

El conocimiento es un activo de carácter continuado en el tiempo que no se deprecia con su utilización ${ }^{1}$. En virtud de esta continuidad, muchos de los avances que se desarrollan en un área específica de la técnica pueden a recaer sobre teorías, descubrimientos o invenciones ya existentes, dando lugar a la denominada "innovación acumulativa"2.

Este escenario, donde la consecución de un resultado depende de otro previo ${ }^{3}$, se muestra particularmente controvertido cuando el conocimiento que sirve de base -en nuestro caso, en forma de invención- se encuentra protegido por un derecho de exclusiva, en concreto, por una patente. Esto es así porque el titular de este derecho puede impedir a cualquier tercero siempre que no medie consentimiento- una serie de actuaciones ${ }^{4}$, como pueda ser la fabricación, el ofrecimiento, la introducción en el comercio e incluso la utilización, importación o posesión para alguno de los fines mencionados del producto o procedimiento objeto de patente ${ }^{5}$. Salvo que se trate de actos realizados en el ámbito privado y con fines no comerciales, de actos realizados con fines experimentales, de estudios y ensayos realizados para la autorización de medicamentos genéricos en España o fuera de España ${ }^{6}$, o de la preparación de medicamentos realizada en las farmacias extemporáneamente y por unidad en ejecución de una receta médica ${ }^{7}$, todo tercero

\footnotetext{
${ }^{1}$ En este sentido, ENCAOUA, D., DOMINIQUE, G y MARTÍNEZ, C., "Sistema de Patentes para fomentar la innovación: lecciones de análisis económico", en Instituto de Políticas y Bienes Públicos (IPP), CCHS-CSIC, Documento de Trabajo núm. 15, Madrid, 2010. pp. 1-39, pp. 5 y 6. http://ipp.csic.es/sites/default/files/content/workpaper/2010/15_encaoua_guellec_martinez.pdf (Consultado el 2 de abril de 2018).

${ }^{2}$ Uno de los primeros artículos científicos que trata y promueve la innovación acumulativa es SCOTCHMER, S, "Standing on Shoulders of Giants: Cumulative Research and the Patent Law", en Journal of Economic Perspectives, vol. 5, 1991, pp. 29-41.

${ }^{3}$ Igualmente se pronunció Isaac Newton en una carta remitida a Robert Hooke en 1676, donde escribió: "If I have seen further, it is by standing upon the shoulders of giants" ("si he visto más allá es porque estoy sentado sobre los hombros de gigantes"). En esta misma línea, Thomas Alva Edison pronunció la siguiente frase: "I start where the last man left off" ("He comenzado donde el último hombre lo dejó") contenida en UNTERMYER, L., Makers of the Modern World: The Lives of Ninety-two Writers, Artists, Scientists, Statesmen, Inventors, Philosophers, Composers, and Other Creators who Formed the Pattern of Our Century, Ed. Simon and Schuster, Nueva York, 1955, p. 227. Ambas realidades son representativas del carácter acumulativo del conocimiento.

${ }^{4}$ Véase el art 59.1 de la Ley 24/2015, de 24 de julio, de Patentes (LP).

${ }^{5}$ Estos actos se consideran de "explotación directa" según la clasificación realizada por BOTANA AGRA, M., "Efectos de la patente", en AA.VV., Manual de la Propiedad Industrial (Dir. FERNÁNDEZ NOVOA. C., OTERO LASTRES, J.M., BOTANA AGRA, M.), Ed. Marcial Pons, Madrid. 2009, p. 161. Esta clasificación ha sido incorporada en la vigente LP que, a diferencia de la normativa anterior en este ámbito, dota a los diferentes preceptos de títulos descriptivos. En este sentido, véanse los arts. 59 y 60 LP.

6 A este límite se le conoce como "cláusula bolar". Según éste no se considera violación del derecho de patente la realización con fines experimentales de estudios y ensayos necesarios para la autorización de medicamentos genéricos. Esta disposición está pensada particularmente para las empresas que pretenden comercializar medicamentos, basándose en un principio activo protegido, esperando al momento en que expire la patente. Para ello deben desarrollar toda una serie de actos preparatorios para conseguir la autorización sanitaria correspondiente y deben probar la eficacia del medicamento para constatar que los efectos son los mismos que el original (pruebas de bioequivalencia). Todas estas actuaciones llevan su tiempo, de ahí que las empresas comiencen dichos trámites antes de que haya finalizado el período de vigencia de la patente. Por ello, el legislador ha precisado la posibilidad de realizar esas pruebas sin entenderlas como un supuesto de infracción de la patente. A mayor abundamiento, sobre la cláusula bolar y sus implicaciones véase, GARCÍA VIDAL, A., "La cláusula Bolar. La licitud de los actos preparatorios de la autorización para comercializar un medicamento genérico realizados durante la vigencia de la correspondiente patente farmacéutica", en ADI, Tomo 26, 2005-2006, pp. 187-206; VIDAL-QUADRAS TRIAS DE BES, M., M, Patentes e industria farmacéutica, Ed. J. M. Bosch, Barcelona, 2006; PONS DE VALL ALOMAR, M., "Los límites de las patentes farmacéuticas", en Anuario Facultad de Derecho de la Universidad de Alcalá, 2008, pp. 273-288; entre otras.

7 También conforman un límite al derecho de patente el empleo de la invención en buques u otros medios de locomoción de países de la Unión de París cuando penetren en España de manera accidental así como los actos Revista de Direito Brasileira | São Paulo, SP | v. 19 | n. 8 | p. x- x |Jan./Abr. 2018
} 
interesado en la invención objeto de patente deberá solicitar autorización a su titular para poder ponerla en práctica.

Es precisamente en este contexto donde las denominadas licencias cruzadas cobran relevancia, ya que se presentan como una posible alternativa a la explotación para el titular de una patente, permitiendo a su vez al tercero interesado explotar su invención dependiente de aquélla. Será necesario, por tanto, delimitar conceptualmente esta realidad así como su configuración y alcance en el ordenamiento español, sin olvidar los posibles efectos que pueda causar en la competencia.

\section{CONCEPTO Y FUNDAMENTO DE LAS LICENCIAS CRUZADAS}

La expresión licencias cruzadas ${ }^{8}$ o "cross-licensing" proviene del mundo anglosajón y hace referencia a aquellos acuerdos, normalmente bilaterales, mediante los cuales las partes implicadas pretenden evitar litigios por infracción de patentes ${ }^{9}$. Como se aprecia, la doctrina anglosajona plantea esta definición desde una perspectiva negativa. De partida, esta concepción no tendría cabida en ordenamiento español ya que, de lo contrario, la literalidad de la misma admitiría la posibilidad de infringir la patente de otro titular -evitando a través de esta fórmula los litigios ocasionados por dicha infracción-. Sin embargo, el ordenamiento jurídico español acoge esta institución jurídica tan sólo si se contempla desde un prisma positivo, esto es, refiriéndonos a ella como aquel acuerdo bilateral en el que ambas partes implicadas se conceden, de manera recíproca, licencia sobre sus respectivas patentes.

La licencia es un medio que permite a un tercero explotar una patente ajena, normalmente mediando consentimiento de su titular. Este último matiz se debe a que existen dos categorías de licencia de patente en el ordenamiento jurídico español, en concreto, la licencia obligatoria y la licencia contractual ${ }^{10}$. Si bien para esta última rige el principio de autonomía de la voluntad de las partes, para el caso de la licencia obligatoria no será necesario el consentimiento del titular de la patente, ya que tiene lugar bajo una serie de causas tasadas que, de manera

contemplados en el art 27 del Convenio de 7 de Diciembre de 1944, relativo a la aviación civil internacional. Éstos y los citados expresamente en el cuerpo del trabajo se encuentran recogidos en el art. 61 LP.

${ }^{8}$ En el ámbito europeo, se utiliza la expresión "acuerdo recíproco" para referirse a esta realidad en sentido amplio. En concreto, el art. 1 d) del Reglamento (UE) 316/2014 de la Comisión, de 21 de marzo de 2014, relativo a la aplicación del artículo 101, apartado 3, del Tratado de Funcionamiento de la Unión Europea a determinadas categorías de acuerdos de transferencia de tecnología (RECATT), utiliza esta denominación cuando se trata de un acuerdo de transferencia de tecnología por el cual dos empresas se conceden recíprocamente una licencia de derechos de tecnología referida a tecnología competidoras.

${ }^{9}$ THE UNITED STATES DEPARTMENT OF JUSTICE, Chapter 3: Antitrust Analysis of Portfolio Cross-Licensing Agreements and Patent Pools, 2014, pp. 57-85, p. 59, disponible en https://www.justice.gov/sites/default/files/atr/legacy/2014/05/30/chapter_3.pdf (Consultado el 27 de marzo de 2018).

${ }^{10}$ Cabría apuntar un tercer grupo de licencias denominadas de pleno derecho, consideradas una especialidad dentro de la licencia contractual disponiendo de un régimen específico (Título VIII, Capítulo III, arts. 87, 88 y 89 LP). Algún autor habla directamente de tres grupos separados; en concreto, BAYLOS CORROZA, H., "Cesión, licencias contractuales y licencias de pleno derecho", en Jornadas sobre la nueva Ley española de Patentes, presentadas los días 14 y 15 de mayo de 1986, Barcelona, Ed. Asociación Internacional para la Protección de la Propiedad Industrial, Barcelona, 1987, pp. 15-32, pp. 29 y ss. En cualquier caso, la licencia de pleno derecho se caracteriza fundamentalmente porque existe un ofrecimiento de licencias por parte del titular de la patente (a través de la OEPM), previo al acuerdo contractual establecido entre el titular de la patente y el que va a ser licenciatario. Se trata de una declaración en la que el titular de la patente está dispuesto a autorizar la utilización de la invención a cualquier interesado. Sobre este extremo véase, BOTANA AGRA, M., "La patente como objeto del derecho de propiedad", en AA.VV., Manual de la Propiedad Industrial (Dir. FERNÁNDEZ NOVOA. C., OTERO LASTRES, J.M., BOTANA AGRA, M.), Ed. Marcial Pons, Madrid. 2009, p. 195.

Revista de Direito Brasileira | São Paulo, SP | v. 19 | n. 8 | p. x- x |Jan./Abr. 2018 
expresa, ha previsto el legislador, por lo que no se configura como medio de explotación voluntario ${ }^{11}$.

Con independencia de su categoría, el rasgo distintivo del fenómeno que nos ocupalicencias cruzadas- radicaría en la exigencia de reciprocidad, ya que el licenciatario como mínimo concede al licenciante una licencia sobre su patente en contraprestación a la licencia obtenida $^{12}$. Esta característica implica una correspondencia mutua de ambas partes implicadas ${ }^{13}$, siendo por ello relevante el motivo que conduce a esta exigencia.

La principal razón de ser de este mecanismo es la dependencia entre patentes. Así, el desarrollo de una tecnología determinada podría precisar la explotación de una patente ajena requiriendo, por tanto, la necesaria concesión de una licencia sobre la misma. En contraprestación, el titular de la referida patente tendría la posibilidad de introducir en el acuerdo de licencia una cláusula que garantice la obtención de una licencia sobre la nueva invención que se desarrolle (licencia recíproca). A través de esta figura se persigue, no sólo el intercambio entre patentes y conocimientos ya existentes, sino también facilitar el tráfico de posibles futuras innovaciones. En este sentido, las partes podrían continuar desarrollando y mejorando la tecnología licenciada y después optar por la consiguiente licencia sobre las nuevas invenciones protegidas $^{14}$. En todo caso, para evitar un posible abuso, estas licencias deberían ir referidas a un área de la práctica concreta, además de ser concedidas por un período de tiempo limitado ${ }^{15}$.

Asimismo, esta reciprocidad característica de las licencias cruzadas se presenta como posible solución a los llamados "patent thickets" -que desarrollaremos infra-, proporcionando la llamada "patent peace", dotando de seguridad jurídica escenarios con una alta concentración de patentes y ámbitos de la técnica emergentes o todavía sin explorar ${ }^{16}$.

\section{SUPUESTOS DE LICENCIAS CRUZADAS CONTEMPLADOS EN LA LEY 24/2015, DE 24 DE JULIO, DE PATENTES}

Existen, pues, dos posibles escenarios en los que las partes pueden verse compelidas a otorgarse de manera recíproca licencias sobre sus respectivas patentes. El primer supuesto lo conformaría aquél en el que el legislador exige otorgar licencia obligatoria por razones de dependencia, situación que se debe fundamentalmente a la existencia de una patente dependiente de otra previa. El segundo supuesto se enmarcaría dentro de la esfera contractual, y se referiría a la situación en que las partes voluntariamente convienen concederse una licencia recíproca, debiendo prestar especial atención al caso de las "patent pools".

\subsection{Licencia obligatoria por razones de dependencia. Posibilidad de cross-licensing}

Las licencias obligatorias suponen un límite al derecho de exclusiva otorgado por la patente a su titular. Las razones que respaldan esta excepción son muy variadas. Así, puede

\footnotetext{
11 Sobre esta categoría, puede consultarse BERCOVITZ RODRÍGUEZ-CANO, A., "Notas sobre las licencias obligatorias de patentes", en $A D I$, núm. 20, 1999, pp. 55-64; LOIS BASTIDA, F., "Las licencias obligatorias de patentes farmacéuticas por parte de países menos desarrollados", Actas de Derecho Industrial, núm. 26, 2005-2006, pp. 495-516. Desde un punto de vista comparado, DE LAS HERAS LORENZO, T., "Las licencias obligatorias en la vigente Ley francesa de Patentes", en Actas de Derecho Industrial, núm. 3, 1976, pp. 157-212.

${ }^{12}$ En este sentido, véase, PERLEGOS, P., "Cross Licensing", en Anti Essays, 3 de marzo de 2011, p. 1. Disponible en http://www.antiessays.com/free-essays/75413.html (Consultado el 13 de abril de 2018).

13 Así, la Primera acepción del vocablo "reciprocidad" de la Real Academia Española de la Lengua.

${ }^{14}$ PERLEGOS, P., “Cross Licensing”, cit., p. 8.

15 GRINDLEY, P.C., y TEECE, D.J., "Managing Intellectual Capital: Licensing and Cross-Licensing in Semiconductors and Electronics”, en California Management Review, vol. 39, núm. 2, 1997, pp. 8-41, pp. 8 y ss.

${ }^{16}$ En este sentido, véase MILGRIM, R. M., en Milgrim's guide to IP licensing, Ed. Wolters Kluwer, New York, 2012, p. 66, según el cual los acuerdos de licencias cruzadas suponen un relativo "margen de maniobra" ("breathing room") para aquellas industrias sobresaturadas con una ingente cantidad de patentes.
} 
perseguir la búsqueda de un equilibrio entre intereses -interés particular/intereses de terceros/interés público-, el impedimento del abuso que pudiera surgir por un potencial monopolio, o bien promover el interés público en sentido amplio -desarrollo de la economía y bienestar social, salud pública ${ }^{17}$ o defensa nacional, entre otras $-{ }^{18}$. Uno de los motivos que subyace de los anteriores como fundamento de las licencias obligatorias es, por tanto, el avance en el progreso científico y tecnológico. Dado que en dicho proceso puede participar cualquier entidad o individuo, no debería suponer obstáculo alguno que los avances anteriores se encuentren protegidos y bajo la esfera de aquel sujeto que los obtuvo. No obstante, si bien protegido, el conocimiento ha de estar disponible, no sólo para proceder a su explotación, sino también para seguir profundizando en él y encontrar otras soluciones a problemas prácticos actuales. Precisamente en este contexto suelen darse situaciones en las que, para continuar con el desarrollo de una nueva invención y sobre todo, para poder explotarla, sea necesario acudir a una anterior que está protegida por una patente.

En España se contempla la dependencia entre patentes ${ }^{19}$ como uno de los supuestos de concesión de licencia obligatoria $(\operatorname{art} 91 \text { b) LP })^{20}$. Se entiende que una patente va a ser dependiente de otra cuando, para la explotación de la primera, sea necesario obtener una licencia por parte del titular de la segunda patente que recaiga sobre esta última. Esto ocurre cuando las partes posean tecnologías jurídicamente protegidas que se encuentran en una posición unilateral o bilateral de bloqueo (blocking patents). Hablaremos de posición de bloqueo unilateral cuando una tecnología patentada requiera para su utilización la obtención de una licencia sobre otra invención que se encuentre protegida mediante patente. Del mismo modo, se dará una situación bilateral de bloqueo cuando la explotación de dos patentes de manera separada requiera la consecución de autorizaciones recíprocas sobre las patentes de las cuales dependen.

El legislador español tan sólo ha previsto la primera situación, en concreto, el art. 65 LP regula de manera expresa esta posición unilateral de bloqueo concediéndole la categoría de patentes dependientes. Así, el hecho de que el objeto de una patente no pueda ser explotado sin utilizar la invención protegida por un título anterior perteneciente a distinto titular no será obstáculo para la validez de aquélla. Ni el titular de la patente anterior podrá explotar la patente posterior durante la vigencia de ésta sin consentimiento de su titular, ni el titular de la patente posterior podrá explotar ninguna de las dos patentes durante la vigencia del derecho anterior, a no ser que cuente con el consentimiento del titular del mismo o haya obtenido una licencia obligatoria.

\footnotetext{
${ }^{17}$ Cuando la concesión de licencias obligatorias está ligada a la salvaguarda del derecho a la salud, normalmente se materializará en el ámbito de las patentes médicas y farmacéuticas en países en vías de desarrollo. A título ilustrativo véase AYODEJI OWOEYE, O., "Compulsory licensing and local drug manufacturing capacity in Africa", en Bulletin of the World Health Organization, vol. 92, núm. 2, 2014, pp. 214-219; KLEIN VIEIRA, L., "Las licencias obligatorias para las patentes de medicamentos: la experiencia brasileña", en Revista de Derecho Económico Internacional, vol. 1, núm. 2, 2011, pp. 25-47, entre otras.

${ }^{18}$ A mayor abundamiento, sobre todos estos motivos y cómo son acogidos por sus diferentes Estados miembros, véase ORGANIZACIÓN MUNDIAL DE LA PROPIEDAD INTELECTUAL, Excepciones y limitaciones a los derechos conferidos por las patentes: licencias obligatorias y/o explotación por el Gobierno (Parte I), Comité Permanente sobre el Derecho de Patentes, Ginebra, 2014. Disponible en http://www.wipo.int/edocs/mdocs/scp/es/scp_21/scp_21_5_rev.pdf (Consultado el 12 de abril de 2018).

${ }_{19}$ A pesar de que en el cuerpo del texto nos refiramos exclusivamente a patentes, cabe señalar que el régimen de licencias obligatorias contenido en la LP resulta aplicable, asimismo, a aquellos casos en los que existe dependencia entre patentes y derechos de obtención vegetal.

${ }^{20}$ También se procederá a la concesión de licencias obligatorias en los siguientes supuestos: ante la falta o insuficiencia de explotación de la invención patentada; ante la necesidad de poner término a prácticas que una decisión administrativa firme de alcance nacional o comunitario, o una sentencia haya declarado contrarias a la legislación de defensa de la competencia; cuando existan motivos de interés público para su concesión; o cuando se trate de la fabricación de medicamentos destinados a la exportación a países con problemas de salud pública previstas en el Reglamento (CE) núm. 816/2006, del Parlamento Europeo y del Consejo, de 17 de mayo de 2006 que las regula (art. $91 \mathrm{LP})$.
} 
Para acudir a este mecanismo -licencia obligatoria- se requiere que el solicitante de la misma demuestre que la invención representa un progreso técnico significativo de considerable importancia económica con relación a la invención reivindicada en la patente anterior (art. 93.4.a LP). De este precepto se desprende el ánimo del legislador de preservar y promover la innovación ya que permite que la invención ulterior pueda ser objeto de patente, aún sabiendo que su explotación precisa de una licencia sobre una patente anterior, o al menos ya contemplada en el estado de la técnica -incluso en forma de solicitud de patente ${ }^{21}-$ Y qué duda cabe que, si se permite la patentabilidad de una invención, se va a tener que garantizar su explotación ya que, por mor del art 90 LP, el titular de la patente está obligado a explotar la invención bien por sí o por persona autorizada (de ahí la licencia obligatoria).

Como contrapartida, es lógico pensar que el titular de la patente anterior deba ser resarcido por dicha obligación. Por ello el legislador ha previsto la posibilidad de que éste pueda solicitar una licencia recíproca sobre la patente dependiente (art. 93.5 LP). Esta medida, si bien a nuestro juicio es acertada, no se encuentra planteada en un plano de igualdad. De un lado, al titular de la patente dependiente se le reconoce el derecho de solicitar la concesión de una licencia obligatoria sobre la patente originaria de la cual depende, sin embargo, al licenciante tan sólo se le ofrece la posibilidad de solicitar una licencia recíproca sobre la patente dependiente, pero ésta quedará circunscrita y en todo caso, obedecerá a la buena voluntad del titular de la patente dependiente, ya que no queda contemplado como una obligación expresa para el licenciatario. Esta intención del legislador puede ser justificable en la medida en que el titular de la patente anterior ha podido y podrá continuar explotando su derecho de exclusiva sin necesidad de solicitar autorización; sin embargo, en el caso del beneficiario de la licencia obligatoria por dependencia, habrá de acudir necesariamente a dicho mecanismo para poder cumplir con su obligación de explotar. De ahí se deduce que el fin buscado sea la protección de la parte más débil de la relación.

En todo caso, el licenciante tiene la posibilidad de limitar el contenido de la licencia, ya que ha de versar sobre lo estrictamente necesario para la explotación de la invención protegida por la patente (art. 93.6 LP). Esto permitirá al licenciatario cumplir con su obligación de explotar evitando, a su vez, su posible comportamiento como free-rider sobre aquellos conocimientos que, dentro del área técnica concreta, excederían de lo rigurosamente imprescindible para cumplir con su deber. Con este mismo fin, la licencia obligatoria carecerá de carácter exclusivo ${ }^{22}$, esto es, el licenciante puede conceder licencias sobre la misma patente a terceros. Sin embargo, por su parte, la licencia obtenida de manera recíproca podría contener una cláusula de exclusividad, aunque, a nuestro juicio, esto es difícil que ocurra en la práctica ya que se somete a la voluntad de ambas partes.

De otro lado, cabe señalar que la licencia recíproca, o en nuestro caso cruzada, debe otorgarse en condiciones razonables (así lo contempla el art. 93.5 LP). Se podría pensar que, al justificar en su solicitud -el titular de la patente anterior- unos términos y condiciones razonables por los que debería concedérsele la licencia sobre la patente posterior, el titular de esta última tan sólo se podría negar a su concesión si esgrimiese unos motivos justos y razonables que sostuviesen su negativa. A nuestro juicio, pues, cabría deducir que el legislador ha introducido este término para, en cierto modo, garantizarle al primer licenciante la posibilidad de obtener una licencia recíproca sobre la patente dependiente, para compensar su carga, y esto lo ha querido

\footnotetext{
${ }^{21}$ Tal y como pone de manifiesto el art. 6.2 y 3 LP, "el estado de la técnica está constituido por todo lo que antes de la fecha de presentación de la solicitud de patente se ha hecho accesible al público en España o en el extranjero por una descripción escrita u oral, por una utilización o por cualquier otro medio". Este precepto entiende igualmente comprendido en el estado de la técnica "el contenido de las solicitudes españolas de patentes o de modelos de utilidad, de solicitudes de patentes europeas que designen a España y de solicitudes de patente internacionales PCT que hayan entrado en fase nacional en España (...)”.

${ }_{22}$ Así lo estipula el art 100.1 LP para todas las licencias obligatorias, con independencia de su causa.
} 
hacer sin imponer una obligación legal al titular de la patente dependiente, pero sí incitándolo a justificar su negativa en caso de que no accediese a la concesión de la licencia.

Cabe destacar también que el régimen contemplado para ambos tipos de licencias es diferente. Mientras que la licencia obligatoria por dependencia se regirá por los términos tanto procedimentales como sustantivos establecidos en la LP (arts. 97 y ss LP), la licencia cruzada obtenida como contrapartida se estipulará basándose en el principio de autonomía de la $\operatorname{voluntad}^{23}$.

Por último y como se ha señalado supra, no existe una regulación expresa de una posible posición de bloqueo bilateral, situación que se da cuando existe una dependencia mutua entre patentes. A priori, podríamos interpretar que resulta aplicable, por analogía, el régimen de las licencias obligatorias a esta realidad. Sin embargo, nuestra regulación en materia de patentes dependientes actúa en forma de cascada -tan sólo tiene cabida cuando de una patente previa depende otra posterior, de tal manera que para su explotación se deba conceder una licencia que recaiga sobre la primera para así cerrar el ciclo-. Esta laguna normativa hace que sea necesario acudir a la autonomía de la voluntad y, por tanto, al régimen de la licencia contractual cuando los titulares de ambos derechos de exclusiva necesiten recíprocamente una licencia sobre sus respectivos títulos. Esto, además, parece estar en consonancia con el requisito previo que exige la LP en virtud del cual, antes de solicitar una licencia obligatoria por razones de dependencia, el licenciatario debe haber intentado, sin éxito, obtener del titular de la patente una licencia contractual (art. 93.4.b LP), figura que se desarrollará a continuación.

\subsection{Acuerdos voluntarios de cross-licensing. Especial atención a los acuerdos "pool" de patente}

Estos acuerdos, al ser voluntarios, quedan bajo la esfera de la autonomía de la voluntad, esto es, las partes pueden estipular en el contrato las cláusulas que consideren oportunas ${ }^{24}$. Se trata, pues, de contratos recíprocos de licencia de patente ${ }^{25}$. Tomados de manera individual, en cada contrato de licencia "una de las partes (licenciante) autoriza a un tercero (licenciatario) a ejercitar todos o algunos de los derechos que se derivan de la patente" ${ }^{\text {26 }}$. Normalmente se otorga un derecho de disfrute sobre la patente que se traduce en un "derecho positivo a la explotación de

\footnotetext{
${ }^{23}$ Este diferente tratamiento se puede manifestar en diferentes escenarios creando cierta inseguridad en relación a los diversos efectos que comportan. A título ilustrativo y en lo que se refiere a la nulidad o caducidad de alguno de los títulos, la LP determina que la licencia obligatoria por dependencia va a quedar sin efecto cuando caduque o sea nulo alguno de los títulos de dicha relación, sin embargo no se estipula nada con respecto a la posible licencia contractual suscitada en consecuencia de la posibilidad contemplada por el legislador al respecto.

${ }^{24}$ Acudiendo a las normas generales de regulación de los contratos, esto es, al Código Civil español, el art. 1255 nos dice que "los contratantes pueden establecer los pactos, cláusulas y condiciones que tengan por conveniente, siempre que no sean contrarios a las leyes, a la moral ni al orden público".

${ }^{25}$ En general, sobre la licencia contractual de derechos de Propiedad Industrial, véanse RONCERO SÁNCHEZ, A., El contrato de licencia de marca, Ed. Civitas, Madrid, 1999; ORTUÑO BAEZA, M. T., "Contratos ligados a la propiedad industrial, licencia de marca: Franquicia”, en AA.VV., Contratos internacionales (Coord. BLANCOMORALES LIMONES, P.), Ed. Tecnos, Madrid, 1997, pp. 1505-1568; MASSAGUER FUENTES, J., El Contrato de Licencia de Know-How, Ed. Bosch, Barcelona, 1989; SEGURA MENA, R.E., "El contrato de know how y licencia del know how”, en El Foro, núm. 12, 2012, pp. 46-58, entre otros. En especial, MARTÍN ARESTI, P., La licencia contractual de patente, Ed. Aranzadi, Pamplona, 1997; MARTÍN ARESTI, P., "Cesión y licencia de patente y marca”, en AA.VV., Contratos Mercantiles, Vol. II (Dirs. BERCOVITZ RODRÍGUEZ-CANO, A y CALZADA CONDE, M.A.), $3^{\text {a }}$ edición, Ed. Thomson Aranzadi, Pamplona, 2007, pp. 2096-2206; o BERCOVITZ ÁLVAREZ, R., en BERCOVITZ ÁlVAREZ, R. y BERCOVITZ ÁLVAREZ, G., "Contratos sobre propiedad industrial”, en AA.VV., Tratado de Contratos, Tomo IV (Dir. BERCOVITZ RODRÍGUEZ-CANO, R.), Ed. Tirant lo Blanch Tratados, $2^{\text {a }}$ Edición, Valencia, 2013, pp. 5238-5320; entre otros.

${ }^{26}$ Así define el contrato de licencia de patente GÓMEZ SEGADE, J. A., y FERNÁNDEZ NOVOA, C., La modernización del Derecho actual de patentes. Ed. Montecorvo, Madrid, 1984. p. 256.
}

Revista de Direito Brasileira | São Paulo, SP | v. 19 | n. 8 | p. x- x |Jan./Abr. 2018 
la invención que la patente protege, con el alcance contractualmente establecido y a cambio de una contraprestación" ${ }^{27}$. Así, con carácter general, podrán ser objeto de acuerdo condiciones tales como el pago de royalties $^{28}$, el carácter exclusivo o no exclusivo de la licencia, o incluso la posibilidad de futuras licencias sobre desarrollos posteriores de la patente, entre otras ${ }^{29}$.

Tal y como se ha mencionado supra, una de las principales razones para establecer acuerdos voluntarios de licencias cruzadas es la existencia de una posición de bloqueo bilateral en el mercado de referencia. Asimismo, se acudirá a este mecanismo cuando la consecución de un producto final exija acceder a una cantidad ingente de patentes sobre las materias primas o los procesos de fabricación del mismo -fenómeno conocido como "patent thicket"30 (maraña de patentes)-, ya que en este escenario existe dificultad para saber si la explotación de una patente podría invadir alguna esfera protegida por otra ${ }^{31}$.

Aquí es donde entran en juego los consorcios de patentes, también llamados "patent pools". Se trata de contratos bilaterales o multilaterales entablados por dos o más sujetos que se reputan titulares de patentes con el fin de disponerlas de tal manera que se puedan licenciar, bien recíprocamente, o bien a terceros en forma de bloque y a través de una única licencia (package license) ${ }^{32}$. Normalmente, estos acuerdos se celebran cuando es necesaria la combinación de varios elementos tecnológicos de naturaleza complementaria ${ }^{33}$, cada uno de ellos protegido por su respectiva patente, para obtener un resultado técnico eficiente ${ }^{34}$, mostrándose en este sentido como herramientas pro-competitivas ${ }^{35}$. Cabe afirmar que dos patentes son complementarias si necesitan explotarse mutuamente para obtener un resultado; todo ello en contraposición a las patentes sustitutivas ${ }^{36}$, que protegen invenciones basadas en tecnologías alternativas y son susceptibles de explotación de manera independiente y paralela. Partiendo de esta distinción,

\footnotetext{
${ }^{27}$ Ésta es la definición de MARTÍN ARESTI, P., La licencia contractual de patente, cit., p. 54.

${ }^{28}$ Por lo que respecta al pago de regalías, en este tipo de acuerdos se tienden a conceder las licencias de manera recíproca sin exigir pago alguno, comportando así una mayor facilidad de entrada al mercado a nuevas empresas que, en caso contrario, deberían incurrir en costes de negociación o de inversión. Véase, BEARD, T.R. y KASERMAN, D.L., "Patent Thickets, cross-licensing and antitrust", The Antitrust Bulletin, núm. 47, 2002, p. 354.

${ }^{29} \mathrm{El}$ régimen de las licencias contractuales de encuentra recogido en el art. $83 \mathrm{LP}$.

${ }^{30}$ Sobre esta realidad, en general, véase SHAPIRO, C., "Navigating the patent ticket: cross licensing, patent pools and standard setting", en Innovation Policy and the Economy, Ed. MIT Press, Londres, 2001, pp. 119-150.

${ }^{31}$ Los acuerdos de cross-licensing permiten combatir este tipo de situaciones ya que reducen el riesgo de invasión de una parcela técnica protegida facilitando, a su vez, el acceso a aquellos derechos de exclusiva titularidad de otra entidad. Véase, BEARD, T.R. y KASERMAN, D.L., "Patent thickets, cross-licensing", cit., p.355.

${ }^{32}$ Tomando la definición otorgada por las Directrices de acompañamiento del RECATT, se entenderán como Technology pools: "arragements whereby two or more parties assemble a package of technology which is license not only to contributors to the pool but also to third parties" (acuerdos en los que dos o más partes forman un "paquete tecnológico" en aras de licenciarlo, no sólo entre los integrantes del acuerdo, sino también a terceros).

${ }^{33}$ Asimismo, este tipo de acuerdos es conveniente alcanzarlo cuando la licencia tenga por objeto los componentes de un producto "estandarizado" conformado, entre otros, por elementos tecnológicos esenciales. Este extremo no será tratado en este trabajo ya que es merecedor de un estudio separado.

${ }^{34}$ Véase la WORLD INTELLECTUAL PROPERTY ORGANISATION, Patent pools and antitrust. A comparative analysis, 2014, p.3. Disponible en http://www.wipo.int/export/sites/www/ipcompetition/en/studies/patent_pools_report.pdf (Consultado el 19 de abril de 2018).

${ }^{35}$ En el ámbito europeo, esta práctica es recomendada en sede de tecnologías complementarias ya que a priori no se espera que éstas compitan entre sí evitando con ello problemas en la competencia. Véase, en este sentido, la Comunicación de la Comisión Europea, Directrices relativas a la aplicación del artículo 81 del Tratado CE a los acuerdos de transferencia de tecnología (2004/C 101/02). Disponible en http://eur-lex.europa.eu/legalcontent/ES/TXT/PDF/?uri=CELEX:52004XC0427(01)\&from=EN (Consultado el 20 de abril de 2018).

${ }^{36}$ Las patentes sustitutivas podrían ocasionar problemas de "hold-up" cuando una de las partes del consorcio imponga condiciones desproporcionadas en su participación, abusando con ello de la necesidad de su licencia para obtener el resultado buscado. Con los pools formados por patentes complementarias este problema se evita. Al respecto, ARMILOTTA, M., Technology Pooling Licensing Agreements: Promoting Patent Access through Collaborative IP Mechanisms, Ed. Nomos, Munich, 2010, p. 110.
} 
cabría afirmar que tan sólo las patentes que recaen sobre invenciones complementarias podrían dar lugar a una posición bilateral o multilateral de bloqueo ${ }^{37}$.

Si bien estos acuerdos se basan en la autonomía de la voluntad, cabría señalar algunas recomendaciones que podrían evitar posibles efectos negativos en la competencia ${ }^{38}$. Así, al crear un consorcio de patentes se debería definir previamente el alcance de la licencia en bloque así como el ámbito de la técnica afectado por aquélla; debería versar sobre patentes complementarias; se deberían incluir tan sólo aquellas patentes que se consideren esenciales para la fabricación del producto final; y por lo que respecta al pago o contrapartida de la licencia (grantbacks) ${ }^{39}$ convendría fijar unos límites razonables (v. gr. no exclusividad de la licencia recíproca, si la hubiere $)^{40}$. A título ilustrativo, sobre este último punto, podría estipularse que los titulares de las patentes objeto del acuerdo no pudiesen conceder licencias de manera independiente y separada al propio grupo ${ }^{41}$, lo que parece ir en detrimento del desarrollo tecnológico ya que orientaría la investigación a un área tecnológica concreta -inicialmente establecida en el acuerdo-. Sensu contrario, la permisión de licencias a terceros ajenos al acuerdo incentiva a los titulares de las patentes a desarrollar sus investigaciones en otras áreas que no sean objeto del "pool"42.

Un aspecto que merece especial mención es el tratamiento del know-how en este tipo de licencias. Así, existirán empresas que habrán dedicado parte de sus recursos -tiempo, capital, y mano de obra- a invertir y desarrollar unos conocimientos previos que conforman la base de una invención protegida mediante patente. Con carácter general, el punto de partida es la necesaria puesta a disposición del licenciatario de todos los conocimientos necesarios para explotar la patente licenciada. Sin embargo, en ocasiones, en sede de un acuerdo voluntario de licencia, cabría optar por no proporcionar a la otra parte contractual toda la información relativa a la invención impidiendo, de esta manera, un acceso adecuado a la tecnología licenciada; pasando de este modo, el know-how y los secretos, a ser inaccesibles ${ }^{43}$. Si bien la LP exige poner a disposición del licenciatario los conocimientos técnicos que sean necesarios para la explotación de la patente, permite sin embargo una cierta discrecionalidad cuando añade la expresión "salvo pacto en contrario" (art. 84 LP). Sería lógico pensar entonces que en el ámbito práctico, y puesto que las licencias cruzadas voluntarias normalmente persiguen alianzas estratégicas, las partes se acojan a dicha posibilidad preservando su know-how respectivo ${ }^{44}$.

\footnotetext{
${ }^{37}$ Las tecnologías que por su objeto resulten ser sustitutivas no se consideran un obstáculo para la explotación individual de las mismas.

${ }^{38}$ Estas directrices o posibles pautas de actuación para evitar un comportamiento anticompetitivo a través de los consorcios de patentes se pueden encontrar en BEENEY, G.R., Pro-Competitive Aspects of Intellectual Property Pools: A Proposal for Safe Harbor Provisions, abril, 2002. pp 5 y ss.

${ }^{39}$ Las cláusulas de "contrapartida" (grantback) normalmente recaen sobre una obligación del beneficiario del consorcio -licenciatario- de licenciar sus derechos de exclusiva al resto de integrantes del acuerdo. Véase, BEENEY, G.R., Pro-Competitive, cit., pp. 11 y ss.

${ }^{40} \mathrm{La}$ exclusividad podría ser problemática si se prevé que los miembros del consorcio se comprometan a conceder licencias sobre los futuros desarrollos de sus invenciones, sobre todo cuando sea cuestionable si dicho desarrollo versa sobre una materia objeto del acuerdo o, por el contrario, pertenece a otra esfera de la técnica.

${ }^{41}$ Este tipo de cláusula es predicable de los consorcios integrados por patentes sustitutivas como medida preventiva para evitar la competencia fuera del acuerdo.

${ }^{42}$ LERNER, J., STROJWAS, M. y TIROLE, J.,"The Design of Patent Pools: The Determinants of Licensing Rules". RAND Journal of Economics, vol.38 núm 3 2007. pp. 610-625. p.611 y ss.

${ }^{43}$ Aquí destaca, BEARD, T.R. y KASERMAN, D.L., "Patent thickets, cross-licensing", cit., p.354.

${ }^{44}$ Esto ocurre cuando consideramos el know-how desde un punto de vista estratégico-empresarial. Desde esta óptica cabría definirlo como "todo conocimiento reservado sobre ideas, productos o procedimientos industriales que el empresario, por su valor competitivo para la empresa, desea mantener oculto". Definición otorgada por GÓMEZ SEGADE, J. A., El secreto industrial (know-how). Concepto y protección. Ed. Tecnos, Madrid, 1974, p. 66. En todo caso, a nuestro juicio, sería conveniente distinguir el know-how necesario, que es el que incluye todos los conocimientos necesarios para una adecuada explotación de la patente y know-how accesorio, del know-how adicional, que englobaría aquellos conocimientos que exceden de este fin. Sobre esta categorización, véase

Revista de Direito Brasileira | São Paulo, SP | v. 19 | n. 8 | p. x- x |Jan./Abr. 2018
} 
Pues bien, para limitar y garantizar unas condiciones razonables y un correcto funcionamiento de la licencia, el acuerdo "pool" lleva normalmente aparejada la creación de una entidad ad hoc que gestione el grupo de patentes licenciado en bloque, tratándose bien de un sujeto integrante del consorcio o bien de un tercero; sin embargo, se trata de una práctica voluntaria $^{45}$. Por su parte, tampoco existe una obligación legal de supervisión para el caso de las licencias cruzadas bilaterales, por lo que se deja al buen hacer de las partes.

\section{PRINCIPALES VENTAJAS E INCONVENIENTES DE LOS ACUERDOS VOLUNTARIOS DE CROSS-LICENSING}

La licencia es un instrumento que, a priori, ayuda a extender el conocimiento y propicia la expansión de las nuevas tecnologías de ahí que, en última instancia, vaya a perseguir el desarrollo económico y tecnológico de un país. De hecho, los acuerdos de licencia normalmente tienden a favorecer los efectos pro-competitivos puesto que fomentan la innovación y ayudan a su difusión.

El hecho de que a través de la licencia se autorice a un tercero a explotar, en las condiciones estipuladas, la invención protegida mediante la patente parece dar a entender que se está actuando en un mercado de libre y abierto y en el que, por tanto, no existe restricción de la competencia. Sin embargo, se ha de estar a lo estipulado en dichos contratos de licencia puesto que algunas provisiones podrían ir más allá de los límites de esa competencia ${ }^{46}$.

Con carácter general, los acuerdos de cross-licensing comportan numerosas ventajas tanto para los titulares de patentes licenciadas como para las empresas interesadas en la investigación y desarrollo de nuevas tecnologías. Así, pueden suponer una reducción en los costes de explotación de una patente ya que disminuye el riesgo de vulneración de un derecho de exclusiva ajeno y, con ello, la incursión en costes de litigio. Asimismo, este mecanismo contribuye a reducir la inversión en términos temporales y económicos en el desarrollo de mejoras de una invención ya protegida ${ }^{47}$, ya que al obtener una licencia sobre ésta, la entidad podría optar por destinar sus recursos a otras actividades más rentables ${ }^{48}$. Asimismo, se facilita una comercialización más rápida del producto final, obteniendo como resultado un mercado variable y adaptado a las nuevas necesidades, lo que radica, en última instancia, en la aceleración del proceso de desarrollo e innovación de las empresas ${ }^{49}$.

Por su parte, las patent pools también generan efectos pro-competitivos al integrar tecnologías complementarias, reduciendo con ello los costes de transacción y solucionando, a su

SÁNCHEZ GARCÍA, L., La configuración jurídica de las invenciones universitarias. Análisis a la luz de la Ley 24/2015, de 24 de julio, de Patentes, Ed. Civitas, Madrid,2017, pp. 320 y ss.

${ }^{45}$ En el ámbito de los productos estandarizados (que quedan fuera de nuestro estudio; véase supra, nota 34), en cuya fabricación se precisa explotar patentes esenciales, existen las denominadas Standard Setting Organisation (entidades normativas) que se encargan de regular parcelas técnicas concretas en aras de poner de acuerdo a todos los agentes implicados para fijar una tecnología de referencia y con ello, términos razonables y no discriminatorios para su licencia. Sobre esta realidad, entre otros, véase SCHAFFER, C., "Prohibiting discriminatory cross-licencsing", en Information \& Communications Technology Law, vol. 21, núm. 1, 2012, pp. 91-106, passim.

${ }^{46}$ JONES, A. y SUFRIN, B., EC Competition Law. Text, cases and material. Ed. Oxford University Press. Nueva York, 2008. p. 789.

${ }^{47}$ A este fenómeno se le conoce como "invent around a patent", y consiste en tratar de conseguir variantes de una invención protegida por patente de manera que sea evitable acudir a ella para obtener un producto determinado, buscando para ello alternativas que puedan sustituirla en el proceso de elaboración del mismo.

${ }^{48} \mathrm{Al}$ respecto véase, BEARD, T.R. y KASERMAN, D.L., "Patent Thickets, cross-licensing", cit., p.355. Este ahorro en los costes de desarrollo de posibles "réplicas" de la patente, normalmente, va a ser superior a los posibles royalties a pagar por la obtención de la licencia. En este sentido, cabe señalar que los acuerdos de cross-licensing normalmente se formalizan sin que exista pago de royalties recíprocos (royalty-free basis).

${ }^{49}$ GIURI, P., y TORRISI, S., "Cross-licensing, Cumulative Inventions and Strategic Patenting”, Draft paper for the 5th Annual Conference of the EPIP Association, Maastricht, September, 20-21, 2010. 
vez, situaciones de bloqueo de patentes ${ }^{50}$. Ponen solución, en cierta medida, al riesgo y a la incertidumbre suscitada en casos donde existe una gran cantidad de patentes alrededor de un producto final, eliminando barreras de entrada a nuevas empresas reduciendo el coste en el que éstas deberían incurrir al introducirse en un nuevo mercado.

Pero no todo son bondades, sino que existen determinadas situaciones en las que este tipo de acuerdos puede generar efectos anticompetitivos y restricciones de la libre competencia no aceptables ${ }^{51}$. Así, es evidente que, para las partes que están implicadas en el acuerdo de crosslicensing, establecer un contrato de este tipo supone eliminar barreras de entrada a un determinado mercado, sin embargo, el efecto causado en terceros es justo el contrario ${ }^{52}$.

Asimismo, cuando las licencias cruzadas se otorguen entre titulares de patentes que persiguen la consecución de un producto final, pudiendo éstos desarrollarlo de manera independiente sin necesidad de acudir a una licencia recíproca -alianza entre competidores en el mercado-, podrían ocasionar una posible merma en la competencia, lo cual ocasiona efectos negativos en los consumidores al reducir su capacidad de elección y al experimentar un incremento en los precios finales del producto.

En lo se refiere a la contraprestación en las licencias cruzadas, es frecuente la estipulación de una cantidad fija a pagar por una de las partes como consecuencia de la vulnerabilidad o grado de dependencia de ésta frente a la otra parte del acuerdo -fenómeno que se conoce como "balancing payments" o pago en compensación- ${ }^{53}$. El problema deviene cuando ambas partes acuerdan fijar de una regalía recíproca. Al proceder de esta manera $-v$. gr. pago de una cantidad por unidad fabricada-, ambos licenciatarios podrían ver incrementado el coste de explotación de su invención, lo que finalmente repercutiría en el precio del producto; situación que se ve agravada cuando ambas invenciones se hayan de combinar para obtener el producto referido, puesto que éste debe soportar los costes marginales de cada uno de ellos que queda materializado en una subida de precios ${ }^{54}$.

Éstos son tan sólo algunos de los efectos positivos y negativos -ventajas e inconvenientes- que puede comportar el establecimiento de acuerdos recíprocos de licencia en la competencia, lo que en definitiva nos conduce a pensar que, en la medida en que éstos se basen en la autonomía de la voluntad, será conveniente que las partes prevean dichos efectos y procuren precisar los límites del acuerdo -pago de regalías, exclusividad, etc- velando por su interés particular, evitando, a su vez, una vulneración de la competencia.

\section{FUENTES}

AYODEJI OWOEYE, O., "Compulsory licensing and local drug manufacturing capacity in Africa", en Bulletin of the World Health Organization, vol. 92, núm. 2, 2014.

ARMILOTTA, M., Technology Pooling Licensing Agreements: Promoting Patent Access through Collaborative IP Mechanisms, Ed. Nomos, Munich, 2010.

\footnotetext{
${ }^{50}$ MORSE, M.H., "Cross-Licensing and Patent Pools: Framework and Practical Issues". ABA Section of Antitrust Law Intellectual Property Comitee Newsletter, Antitrust and Intellectual Property, vol. 3, núm. 1, 2002, pp. $42-53$. p. 45

${ }^{51}$ Así lo sugiere BERCOVITZ RODRÍGUEZ CANO, A., "Introducción a la problemática jurídica de las licencias", en Estudios sobre el futuro Código Mercantil. Libro homenaje al profesor Rafael Illescas Ortiz, Ed. Universidad Carlos III de Madrid, Getafe, 2015, pp.1011-1028, p. 1017.

${ }^{52}$ BEARD, T.R. y KASERMAN, D.L., "Patent Thickets, cross-licensing", cit., p.361.

${ }^{53}$ En este sentido véase, SHAPIRO, C., "Navigating the Patent Thicket", cit., p. 131.

${ }^{54}$ Véase, GILBERT, R. y SHAPIRO, C., "Antitrust Issues in the Licensing of Intellectual Property", Brooking Papers on Economic Activity (Microeconomics), 13, 1997, pp. 283-336. p. 283.
} 
BAYLOS CORROZA, H., "Cesión, licencias contractuales y licencias de pleno derecho", en Jornadas sobre la nueva Ley española de Patentes, presentadas los días 14 y 15 de mayo de 1986, Barcelona, Ed. Asociación Internacional para la Protección de la Propiedad Industrial, Barcelona, 1987.

BEARD, T.R. y KASERMAN, D.L., "Patent Thickets, cross-licensing and antitrust", The Antitrust Bulletin, núm. 47, 2002.

BEENEY, G.R., Pro-Competitive Aspects of Intellectual Property Pools: A Proposal for Safe Harbor Provisions, abril, 2002.

BERCOVITZ ÁLVAREZ, R., en BERCOVITZ ÁLVAREZ, R. y BERCOVITZ ÁLVAREZ, G., "Contratos sobre propiedad industrial", en AA.VV., Tratado de Contratos, Tomo IV (Dir. BERCOVITZ RODRÍGUEZ-CANO, R.), Ed. Tirant lo Blanch Tratados, $2^{\text {a }}$ Edición, Valencia, 2013.

BERCOVITZ RODRÍGUEZ-CANO, A., "Notas sobre las licencias obligatorias de patentes", en Actas de Derecho Industrial, núm. 20, 1999.

— "Introducción a la problemática jurídica de las licencias", en Estudios sobre el futuro Código Mercantil. Libro homenaje al profesor Rafael Illescas Ortiz, Ed. Universidad Carlos III de Madrid, Getafe, 2015.

BOTANA AGRA, M., "La patente como objeto del derecho de propiedad", en AA.VV., Manual de la Propiedad Industrial (Dir. FERNÁNDEZ NOVOA. C., OTERO LASTRES, J.M., BOTANA AGRA, M.), Ed. Marcial Pons, Madrid, 2009.

— "Efectos de la patente", en AA.VV., Manual de la Propiedad Industrial (Dir. FERNÁNDEZ NOVOA. C., OTERO LASTRES, J.M., BOTANA AGRA, M.), Ed. Marcial Pons, Madrid, 2009.

DE LAS HERAS LORENZO, T., "Las licencias obligatorias en la vigente Ley francesa de Patentes”, en Actas de Derecho Industrial, núm. 3, 1976.

ENCAOUA, D., DOMINIQUE, G y MARTÍNEZ, C., "Sistema de Patentes para fomentar la innovación: lecciones de análisis económico", en Instituto de Políticas y Bienes Públicos (IPP), CCHS-CSIC, Documento de Trabajo núm. 15, Madrid, 2010, disponible en http://ipp.csic.es/sites/default/files/content/workpaper/2010/15_encaoua_guellec_martinez.pdf.

GARCÍA VIDAL, A., "La cláusula Bolar. La licitud de los actos preparatorios de la autorización para comercializar un medicamento genérico realizados durante la vigencia de la correspondiente patente farmacéutica", en ADI, Tomo 26, 2005-2006.

GIURI, P., y TORRISI, S., "Cross-licensing, Cumulative Inventions and Strategic Patenting”, Draft paper for the 5th Annual Conference of the EPIP Association, Maastricht, September, 2021, 2010.

GÓMEZ SEGADE, J. A., El secreto industrial (know-how). Concepto y protección. Ed. Tecnos, Madrid, 1974.

— La modernización del Derecho actual de patentes. Ed. Montecorvo, Madrid, 1984. 
GRINDLEY, P.C., y TEECE, D.J., "Managing Intellectual Capital: Licensing and CrossLicensing in Semiconductors and Electronics", en California Management Review, vol. 39, núm. 2, 1997.

GILBERT, R. y SHAPIRO, C., "Antitrust Issues in the Licensing of Intellectual Property", Brooking Papers on Economic Activity (Microeconomics), 13, 1997.

JONES, A. y SUFRIN, B., EC Competition Law. Text, cases and material. Ed. Oxford University Press. Nueva York, 2008.

KLEIN VIEIRA, L., "Las licencias obligatorias para las patentes de medicamentos: la experiencia brasileña”, en Revista de Derecho Económico Internacional, vol. 1, núm. 2, 2011.

LERNER, J., STROJWAS, M. y TIROLE, J.,'The Design of Patent Pools: The Determinants of Licensing Rules". RAND Journal of Economics, vol.38 núm 32007.

LOIS BASTIDA, F., "Las licencias obligatorias de patentes farmacéuticas por parte de países menos desarrollados”, ADI, núm. 26, 2005-2006.

MARTÍN ARESTI, P., La licencia contractual de patente, Ed. Aranzadi, Pamplona, 1997.

- "Cesión y licencia de patente y marca", en AA.VV., Contratos Mercantiles, Vol. II (Dirs. BERCOVITZ RODRÍGUEZ-CANO, A y CALZADA CONDE, M.A.), $3^{\text {a }}$ edición, Ed. Thomson Aranzadi, Pamplona, 2007.

MASSAGUER FUENTES, J., El Contrato de Licencia de Know-How, Ed. Bosch, Barcelona, 1989.

MILGRIM, R. M., en Milgrim's guide to IP licensing, Ed. Wolters Kluwer, New York, 2012.

SCOTCHMER, S, "Standing on Shoulders of Giants: Cumulative Research and the Patent Law", en Journal of Economic Perspectives, vol. 5, 1991.

MORSE, M.H., "Cross-Licensing and Patent Pools: Framework and Practical Issues". ABA Section of Antitrust Law Intellectual Property Comitee Newsletter, Antitrust and Intellectual Property, vol. 3, núm. 1, 2002.

ORTUÑO BAEZA, M. T., "Contratos ligados a la propiedad industrial, licencia de marca: Franquicia”, en AA.VV., Contratos internacionales (Coord. BLANCO-MORALES LIMONES, P.), Ed. Tecnos, Madrid, 1997.

PERLEGOS, P., "Cross Licensing”, en Anti Essays, 3 de marzo de 2011, disponible en http://www.antiessays.com/free-essays/75413.html.

PONS DE VALL ALOMAR, M., "Los límites de las patentes farmacéuticas", en Anuario Facultad de Derecho de la Universidad de Alcalá, 2008.

RONCERO SÁNCHEZ, A., El contrato de licencia de marca, Ed. Civitas, Madrid, 1999. 
SÁNCHEZ GARCÍA, L., La configuración jurídica de las invenciones universitarias. Análisis a la luz de la Ley 24/2015, de 24 de julio, de Patentes, Ed. Civitas, Madrid, 2017.

SCHAFFER, C., "Prohibiting discriminatory cross-licencsing", en Information \& Communications Technology Law, vol. 21, núm. 1, 2012.

SEGURA MENA, R.E., "El contrato de know how y licencia del know how", en El Foro, núm. $12,2012$.

SHAPIRO, C., "Navigating the patent ticket: cross licensing, patent pools and standard setting", en Innovation Policy and the Economy, Ed. MIT Press, Londres, 2001.

UNTERMYER, L., Makers of the Modern World: The Lives of Ninety-two Writers, Artists, Scientists, Statesmen, Inventors, Philosophers, Composers, and Other Creators who Formed the Pattern of Our Century, Ed. Simon and Schuster, Nueva York, 1955.

VIDAL-QUADRAS TRIAS DE BES, M., M, Patentes e industria farmacéutica, Ed. J. M. Bosch, Barcelona, 2006.

ORGANIZACIÓN MUNDIAL DE LA PROPIEDAD INTELECTUAL, Excepciones y limitaciones a los derechos conferidos por las patentes: licencias obligatorias y/o explotación por el Gobierno (Parte I), Comité Permanente sobre el Derecho de Patentes, Ginebra, 2014, disponible en http://www.wipo.int/edocs/mdocs/scp/es/scp_21/scp_21_5_rev.pdf.

THE UNITED STATES DEPARTMENT OF JUSTICE, Chapter 3: Antitrust Analysis of Portfolio Cross-Licensing Agreements and Patent Pools, 2014, disponible en https://www.justice.gov/sites/default/files/atr/legacy/2014/05/30/chapter_3.pdf.

WORLD INTELLECTUAL PROPERTY ORGANISATION, Patent pools and antitrust. A comparative analysis, 2014, disponible en http://www.wipo.int/export/sites/www/ipcompetition/en/studies/patent_pools_report.pdf . 